\title{
Thermally Induced Inversion of the Cholesteric Sense in Lyotropic Polypeptide Liquid Crystals
}

\author{
Hirokazu ToRIUmI, ${ }^{* 1}$ Yoshiko KusUmI, ${ }^{* 1}$ Ichitaro Uematsu, ${ }^{* 1 .}{ }^{\dagger}$ \\ and Yoshiko UEMATSU*2 \\ ${ }^{* 1}$ Department of Polymer Technology, Tokyo Institute of Technology, \\ 2-12-1 Ookayama, Meguro-ku, Tokyo 152, Japan. \\ ${ }^{*}$ Faculty of Engineering, Tokyo Institute of Polytechnics, \\ Iiyama, Atsugi-shi, Kanagawa 243-02, Japan.
}

(Received June 25, 1979)

\begin{abstract}
Thermally induced inversion of the cholesteric sense has been found in lyotropic polypeptide liquid crystals. The cholesteric sense of poly( $\gamma$-benzyl L-glutamate) liquid crystal in $m$ cresol $\left(17 \mathrm{vol}^{\%}\right.$ ) inverts from right to left at $60^{\circ} \mathrm{C}$, and the reciprocal of the cholesteric pitch changes linearly with temperature. The compensated liquid crystalline solution at $60^{\circ} \mathrm{C}$ shows no helical twist. Similar phenomena are found for poly $(\gamma$-benzyl L-glutamate) in benzyl alcohol and for poly $(\gamma$-propyl L-glutamate) in $m$-cresol. The solvent effect on the helical twisting power is discussed in the light of the fact that the side-chain ester groups of poly( $\gamma$-benzyl L-glutamate) form hydrogen bonds with the hydroxyl groups of $m$-cresol.
\end{abstract}

KEY WORDS Polypeptide / Liquid Crystal / Cholesteric Sense / Inversion /

Pitch / Hydrogen Bond / Poly $(\gamma$-benzyl L-glutamate) / $m$-Cresol /

Concentrated solutions of poly $(\gamma$-benzyl L-glutamate) (PBLG) and poly( $\gamma$-alkyl L-glutamate)s (alkyl $=$ methyl, ethyl and propyl) in certain organic solvents form cholesteric liquid crystals. ${ }^{1,2}$ The cholesteric structure in such solutions is characterized by the microscopically visible striation patterns when viewed along a direction normal to the axis of torsion. The distance between striations corresponds to one-half the pitch of the helical structure. It is known that the cholesteric pitch varies with polymer concentration, temperature and solvent. The temperature dependence of the pitch has been investigated in various solvents, such as dichloromethane, chloroform, dichloroethane, benzene, dioxane, and dimethylformamide. ${ }^{3,4}$ In all these systems, the pitch increases with temperature, in contrast to the change in ordinary thermotropic liquid crystals, for which the pitch decreases with temperature. ${ }^{5}$ The positive temperature dependence of the pitch observed for PBLG liquid crystals is usually attributed to the isotropic thermal motion of the PBLG molecule around its long molecular axis,

† To whom correspondence should be addressed. which reduces the anisotropy in the intermolecular potential. ${ }^{6}$

Robinson et al. ${ }^{2}$ have investigated PBLG liquid crystals in a number of solvents, and have shown that the sense of the cholesteric twist depends on the nature of solvent. For example, the PBLG liquid crystal in dioxane forms a right-handed cholesteric structure, while in dichloromethane forms a lefthanded one. In an appropriate solvent mixture of dioxane and dichloromethane (at 0.2 volume fraction of dioxane), the solution shows no regular striation, thus indicating that the cholesteric structure is compensated. A similar compensation phenomenon has been found in a mixture of dioxane and nitrobenzene (at 0.4 volume fraction of dioxane) by DuPre et al., ${ }^{7}$ who also reported that the addition of trifluoroacetic acid to PBLG in dioxane (up to $10 \%$ ) causes a decrease in the pitch of the cholesteric liquid crystal. There are some observations indicating the presence of intermolecular hydrogen bonds between the PBLG side chains and the solvent molecules. Electric dichroism studies show that a small amount of trifluoroacetic acid interacts with the side chain ester group either by hydrogen bonding or pro- 
tonation. ${ }^{8.9}$ Infrared studies show that, in the $m$ cresol solution, hydrogen bonds can be also formed between side-chain ester groups and hydroxyl groups of the solvent. ${ }^{10}$

The purpose of this study is to examine the effect of temperature and polymer-solvent interaction on the helical twisting power of lyotropic polypeptide liquid crystals. ${ }^{11}$ The temperature dependence of the pitch and the thermally-induced inversion of the cholesteric sense are discussed in terms of Keating's theory. ${ }^{12}$ The role of the hydrogen bond in polypeptide liquid crystals is also discussed.

\section{EXPERIMENTAL}

The samples of PBLG and poly( $\gamma$-ethyl L-glutamate) (PELG) were synthesized by the $\mathrm{N}$ carboxyanhydride method using triethylamine as an initiator in dioxane. Average molecular weights were estimated to be $10.0 \times 10^{4}$ for PBLG and $9.0 \times 10^{4}$ for PELG by viscosity measurements. The sample of poly( $\gamma$-methyl L-glutamate) (PMLG) was kindly supplied by Ajinomoto Co. Inc., Japan (Ajicoat A2000). The average molecular weight was about $10.0 \times 10^{4}$. Poly $(\gamma$-propyl L-glutamate) (PPLG) was prepared by the alcoholysis ${ }^{13}$ of PMLG, and the substitution of methyl groups by propyl groups was confirmed by NMR spectra. $m$-Cresol and benzyl alcohol were used as solvents after vacuum distilled.

A solution containing a $17 \%$ volume fraction of polymer was transfered into a glass cell of $2.0 \mathrm{~mm}$ in thickness, which was then hermetically sealed to prevent solvent loss. This solution was matured at room temperature for several weeks to allow formation of the liquid crystalline structure. In order to determine the equilibrium pitch, the solution was maintained at each measurement temperature for more than 10 hours in a thermostated oven. The pitch was measured with a polarizing microscope by the photographic recording method. The optical diffraction technique with a $\mathrm{He}-\mathrm{Ne}$ gas laser was in some cases employed for the pitch measurement.

The optical rotatory dispersion (ORD) was measured with a Shimazu QV-50 spectrometer with ORD attachments. A quartz cell of $0.2 \mathrm{~mm}$ in thickness was mounted on a holder, and both the polarizer and the analyzer were rotated in a plane perpendicular to the incident beam.

The infrared (IR) spectrum was measured by using a JASCO DS-701G infrared spectrophotometer. The solution was interposed between two KRS-5 (thallium bromide-thallium iodide mixed crystal) plates. The variation of the carbonyl ester band with temperature was recorded in the $1700-1750 \mathrm{~cm}^{-1}$ region. Absorption due to solvent was negligibly small in this frequency region.

\section{RESULTS}

Figure 1a shows a series of photographs of the striation patterns of the PBLG liquid crystal in $m$ cresol observed under a polarizing microscope. The distance between striations increases with temperature, starting from about $10 \mu \mathrm{m}$ at $30^{\circ} \mathrm{C}$. At $60^{\circ} \mathrm{C}$, the striation patterns are replaced by threads characteristic of nematic liquid crystals. Above this temperature, the striation patterns appear again, and the distance decreases with temperature. In the high temperature range $\left(130-160^{\circ} \mathrm{C}\right)$, a biphasic (isotropic, liquid crystalline) region appears; the liquid crystalline phase transmits the light, but the isotropic phase does not, when observed between crossed polars. Above $160^{\circ} \mathrm{C}$, only the isotropic phase exists. On cooling such an isotropic solution, a birefringent phase appears first as spherulites with black maltasecrosses. The PBLG molecule has been shown to retain its $\alpha$-helical conformation in $m$-cresol up to $200^{\circ} \mathrm{C} .{ }^{14}$ Therefore, this change should correspond to the high-temperature phase transition observed for the PBLG liquid crystal in dimethylformamide by Wee and Miller. ${ }^{15}$

Figure $1 \mathrm{~b}$ shows the optical diffraction patterns of the liquid crystalline solution of PBLG in $m$-cresol. A one-dimensional array of diffraction spots observed at lower temperatures indicates the presence of regular gratings in the liquid crystalline solution. The diffraction angle $(\theta)$ decreases with temperature in the range below $60^{\circ} \mathrm{C}$. The solution at $60^{\circ} \mathrm{C}$ shows only a diffuse central spot. Above $60^{\circ} \mathrm{C}$, the diffraction angle increases with temperature, but the diffraction pattern changes its shape into a circular one. We obtained the value of the cholesteric half-pitch $(S)$ using the following equation,

$$
2 S \sin \theta=\frac{m \lambda}{n},
$$

where $m$ is the order of diffraction, $n=1.54$ is the refractive index of the medium, and $\lambda=632.8 \mathrm{~nm}$ is the wavelength of the beam from the $\mathrm{He}-\mathrm{Ne}$ gas 

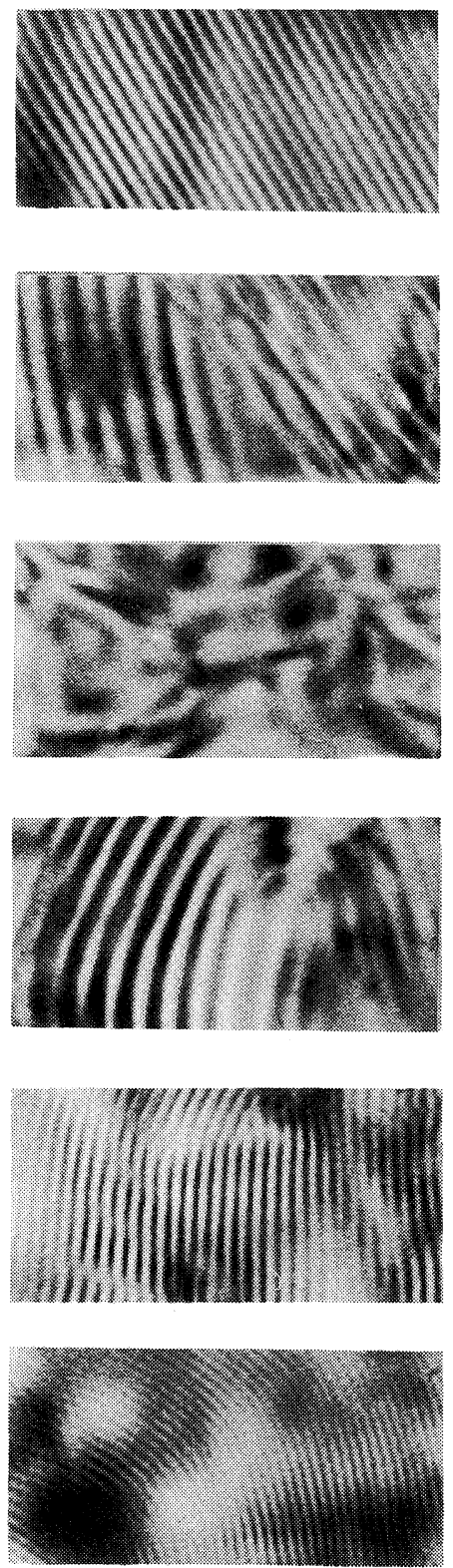

b

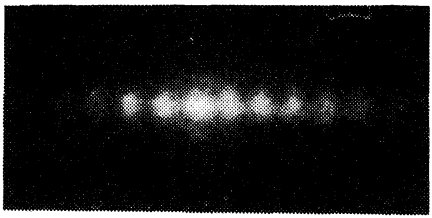

$30^{\circ} \mathrm{C}$

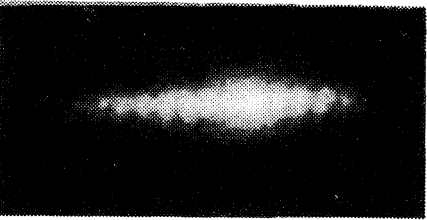

$40^{\circ} \mathrm{C}$

$60^{\circ} \mathrm{C}$

$80^{\circ} \mathrm{C}$

$100^{\circ} \mathrm{C}$

$120^{\circ} \mathrm{C}$

Figure 1. Cholesteric liquid crystalline structure of PBLG in $m$-cresol: (a) striation patterns observed under polarizing microscope; (b) optical diffraction patterns with a beam from the $\mathrm{He}-\mathrm{Ne}$ gas laser. Concentration is $17 \%$ volume fraction of polymer, and cell thickness is $2 \mathrm{~mm}$.

laser. The calculated values are shown in Figure 2, together with the average spacings determined by microscopic observation. Values obtained by these two different methods were found to be in good agreement.

Cholesteric liquid crystals show a very high "form optical rotation" associated with periodicity in the cholesteric structure. When the wavelength of the 


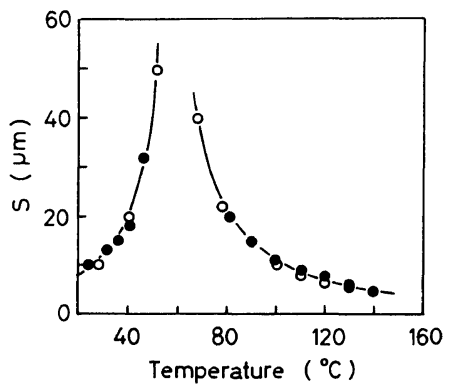

Figure 2. Plot of the half-pitch $v s$. temperature for PBLG liquid crystals in $m$-cresol: $(O)$ microscopic observation; (O) optical diffraction method with the $\mathrm{He}-\mathrm{Ne}$ gas laser.

incident light is shorter than the cholesteric pitch $(P=2 S)$, the sign of the optical rotation is positive for the right-handed cholesteric structure and negative for the left-handed one. According to the modified de Vries theory, ${ }^{16}$ the wavelength dispersion of optical rotatory power $(\Theta)$ can be expressed as follows,

$$
\Theta=\frac{45(\Delta n)^{2} P}{\lambda^{2}} \quad\left(\text { degree } \mu \mathrm{m}^{-1}\right),
$$

where $\Delta n$ is the birefringence. $\Theta$ was measured for six different wavelengths $(\lambda=400,450,500,550,600$ and $650 \mathrm{~nm})$ at several temperatures. By plotting $\Theta$ against $\lambda^{-2}$, straight lines were obtained as shown in Figure 3. The sign of $\Theta$ changes from positive to negative at $60^{\circ} \mathrm{C}$. This is a direct proof for the inversion of the cholesteric sense from right to left. The solution at $60^{\circ} \mathrm{C}$ shows no form optical rotation.

A similar thermally-induced inversion of the cholesteric sense was observed for the PBLG liquid

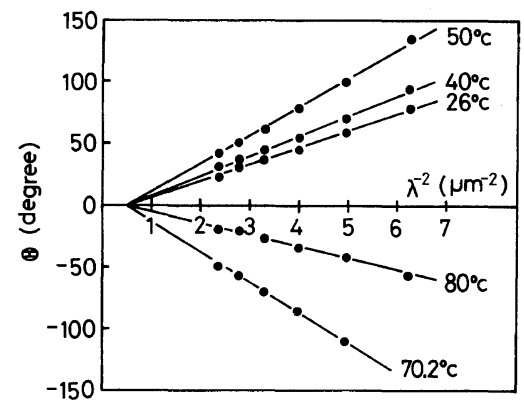

Figure 3. Temperature dependence of the optical rotation of the PBLG liquid crystal in $m$-cresol.

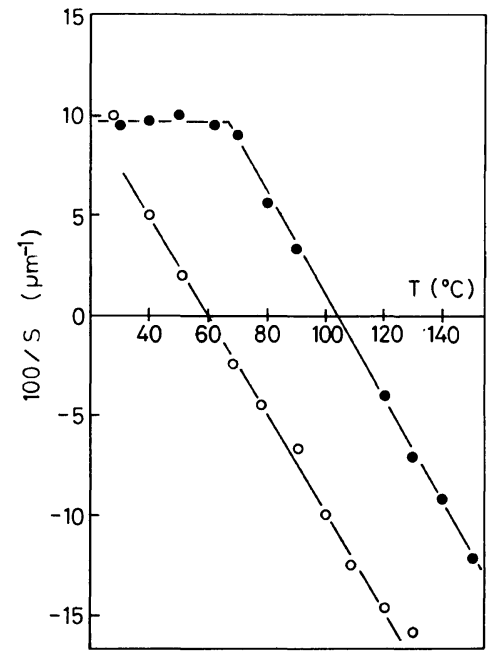

Figure 4. Plot of the reciprocal half-pitch $v s$. temperature for PBLG liquid crystals: $(O)$ in $m$-cresol; (O) in benzyl alcohol.

crystal in benzyl alcohol. In this solution, a gel-like opaque phase coexists with the cholesteric phase at lower temperatures. The opaque phase disappears around $70^{\circ} \mathrm{C}$, where endothermic peaks are observed in the differential scanning calorimetry curve. At the same time, the regularity of the striation patterns becomes better. The value of $S$ below $70^{\circ} \mathrm{C}$ remains constant, and then changes linearly with temperature above $70^{\circ} \mathrm{C}$. The compensation occurs at about $103^{\circ} \mathrm{C}$, and the transition from the biphasic phase to the isotropic phase is observed above $150^{\circ} \mathrm{C}$ in this case. The results are summarized in Figure 4, where the reciprocal of the half-pitch is plotted against temperature. The sign of $1 / S$ is taken as positive when the cholesteric sense is the right-handed. Since the value of $1 / S$ is proportional to the twisting angle $(\phi)$ between neighboring molecular layers, it provides a measure of the helical twisting power. The angle $\phi$ is given by

$$
\phi=180 \frac{d}{S} \quad \text { (degree), }
$$

where $d$ is the distance between molecular layers. For the PBLG liquid crystal in dioxane $(17 \%$ volume fraction of PBLG), Robinson ${ }^{2}$ obtained a value of $d=27 \AA$ by $\mathrm{X}$-ray measurement. Using this value, we calculated $\phi=0.024^{\circ}$ for $1 / S=0.05 \mu \mathrm{m}^{-1}$. The angle changes linearly with temperature $(T)$, and the 
empirical relation is expressed as $\phi=-0.012$ $\left(T-T_{\mathrm{c}}\right)$ for PBLG liquid crystals in $m$-cresol $\left(T_{\mathrm{c}}=60^{\circ} \mathrm{C}\right)$ and in benzyl alcohol $\left(T_{\mathrm{c}}=103^{\circ} \mathrm{C}\right)$.

The temperature dependence of $1 / S$ for PMLG, PELG, and PPLG liquid crystals in $m$-cresol are shown in Figure 5. The PPLG liquid crystal exhibits a compensation at about $67^{\circ} \mathrm{C}$, and the cholesteric sense inverts from right to left. The cholesteric pitches of PMLG and PELG liquid crystals decrease monotonously with temperature. Extrapolation of straight lines indicates that this compensation should occur at 10 and $2^{\circ} \mathrm{C}$, respectively for PMLG and PELG liquid crystals.

In order to examine the polymer-solvent interactions, an IR study was carried out for the PBLG liquid crystal in $m$-cresol. Figure 6 shows the variation of the carbonyl ester band in the 1700-1750$\mathrm{cm}^{-1}$ region with temperature. At $27^{\circ} \mathrm{C}$, the ester band has a peak at $1715 \mathrm{~cm}^{-1}$ and a shoulder at about $1735 \mathrm{~cm}^{-1}$. As the temperature increases, the intensity of the peak at $1735 \mathrm{~cm}^{-1}$ increases while that at $1715 \mathrm{~cm}^{-1}$ decreases. Since formation of the hydrogen bond shifts the carbonyl peak to a lower frequency, ${ }^{10}$ thè peaks at $1735 \mathrm{~cm}^{-1}$ and $1715 \mathrm{~cm}^{-1}$ may be assigned to the carbonyl ester group in the free and hydrogen bonded state, respectively. It appears therefore that a greater portion of the carbonyl ester groups in the $m$-cresol solution are hydrogen-bonded at room temperature. A rough estimate from the relative intensities of the peaks at

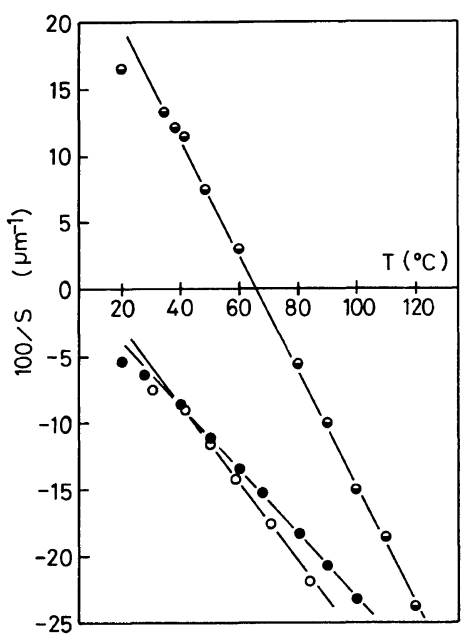

Figure 5. Plot of the reciprocal half-pitch vs. temperature for $\operatorname{poly}(\gamma$-alkyl L-glutamate) liquid crystals in $\mathrm{m}$ cresol: (○) PMLG; (๑) PELG; (Ө) PPLG.

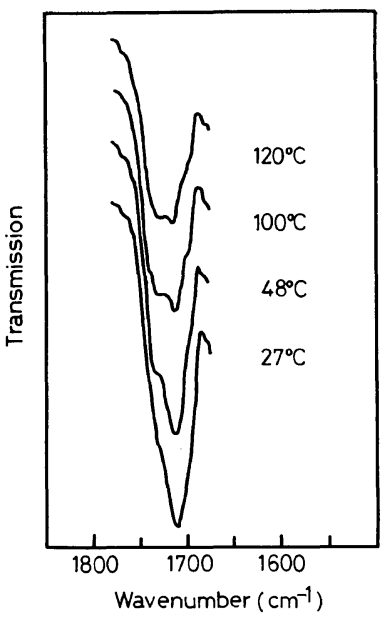

Figure 6. Temperature dependence of IR absorption bands centered at $1715 \mathrm{~cm}^{-1}$ and $1735 \mathrm{~cm}^{-1}$ in the PBLG- $m$-cresol liquid crystal.

$1735 \mathrm{~cm}^{-1}$ and $1715 \mathrm{~cm}^{-1}$ indicates that at $120^{\circ} \mathrm{C}$, almost $1 / 2$ of the original number of hydrogen bonds are still preserved.

\section{DISCUSSION}

\section{Temperature Dependence of the Helical Twisting} Power

The linear dependence of the helical twisting power on temperature is well explained by Keating's theory. ${ }^{12}$ He proposed the theory of the cholesteric twist based on the hindered rotation of molecules due to thermal excitation in a plane normal to the axis of torsion. If we plot the variation of Keating's potential with $\phi$ along the horizontal axis, we get an asymmetrical curve around the vertical axis with a minimum at $\phi=0$. The time-average of the twisting angle does not vanish, because of the anharmonicity in the forces resisting the twist between neighboring planes of molecules. This theory provides a relationship between the average twisting angle $\langle\phi\rangle$ and the temperature,

$$
\langle\phi\rangle=\frac{A k}{2 I \omega^{4}} T,
$$

where $k$ is the Boltzmann constant, $\omega$ is the frequency of the excited twist mode, $I$ is the moment of inertia of the molecule, and $A$ is the constant in the cubic anharmonicity term of the anharmonic equa- 
tion of motion. Equation 4 provides a linear dependence of $\langle\phi\rangle$ on $T$. Keating's theory, however, can not explain the inversion of the cholesteric sense, since the minimum of the intermolecular potential is located at $\phi=0$. A theoretical explanation for the shift in the potential minimum from $\phi=0$ to a finit $\phi_{0}$ was given by Goossens. ${ }^{18}$ According to his theory, the intermolecular potential which is dependent on the angle between two chiral molecules is obtained by calculating the dipole-dipole and dipolequadrupole dispersion energies. A nonzero value for the dispersion energy determined by dipolequadrupole interactions gives rise to the inherent helical twist which is temperature-independent. Here, we attempt a synthesis of Keating's model and the conclusion of Goossens' theory, in order to explain our observations. Suppose an asymmetrical potential curve with the shift of the minimum from $\phi=0$ to $\phi_{0}$ (right-handed). If anharmonicity appears in the opposite direction of the shift (left-handed), the twisting power with the right-handed chirality first decreases with increasing temperature, goes through zero, and then the twisting power begins to increase with the left-handed chirality. Now the compensation is expected to occur at a temperature $T_{\mathrm{c}}$ according to the relationship, $\left(A k / 2 I \omega^{4}\right) T_{\mathrm{c}}=\phi_{0}$. Thus, we obtain the following equation by replacing $T$ in eq 4 with a corrected temperature $T-T_{\mathrm{c}}$ :

$$
\langle\phi\rangle=-\frac{A k}{2 I \omega^{4}}\left(T-T_{\mathrm{c}}\right),
$$

where the negative sign indicates that the cholesteric sense is of the right-handed below $T_{\mathrm{c}}$. The dependence of the twisting power on temperature illustrated in Figure 4 and 5 is well described by this equation.

One can see from Figure 5 that $T_{\mathrm{c}}$ of poly $(\gamma$-alkyl L-glutamate) liquid crystals depends on the sidechain length. ${ }^{17}$ For PMLG and PELG, $T_{\mathrm{c}}$ values are found below room temperature. Consequently, the twisting power of these liquid crystals increases linearly with temperature. On the other hand, if $T_{\mathrm{c}}$ is higher than the temperature range of measurement, this twisting power should decrease with temperature. The observation that the pitches of PBLG liquid crystals in dioxane and in dichloromethane increase with temperature can be understood by this shift of $T_{\mathrm{c}}$.

\section{Solvent Effect on the Cholesteric Sense}

It was shown above that the side chain ester groups of PBLG interacted with $m$-cresol by the formation of hydrogen bonds. In this system, the cholesteric sense was found to be the right-handed at low temperatures. It has been also shown that the cholesteric sense of the PBLG liquid crystal in dichloromethane can be inverted from left to right by the addition of a small amount of ethanol $(10 \%){ }^{2}$ Other evidence for the formation of intermolecular hydrogen bonds has been reported by Bradbury et $a l,{ }^{10}$ who examined the IR spectra of dilute solutions of PBLG in dichloromethane, in $m$-cresol and in a mixture of both solvents. The ester band in the dichloromethane solution shows a single peak at $1735 \mathrm{~cm}^{-1}$. The addition of $m$-cresol changes the shape of the ester band, and the peak at $1715 \mathrm{~cm}^{-1}$ appears. Dichloromethane, which cannot form hydrogen bonds, supports the left-handed cholesteric structure. On the other hand, $m$-cresol supports the right-handed cholesteric structure. These observations may imply the role of hydrogen bonds in determining the cholesteric sense of PBLG liquid crystals.

Milstien and Charney ${ }^{9}$ have already pointed out that hydrogen-bond formation changes the sidechain conformation and the orientation of the permanent dipole moment located on the carbonyl ester group. In the case of the polypeptide liquid crystal, anisotropy in the intermolecular potential strongly depends on the side-chain conformation as well as the backbone $\alpha$-helical conformation. Thus, we expect that the changes in the surface environment of the polymer caused by the hydrogen-bond formation can alter the helical twisting power and lead to the inversion of the cholesteric sense.

In spite of the fact that the PPLG molecule has no aromatic ring, the PPLG liquid crystal in $m$-cresol forms the right-handed cholesteric structure at low temperatures, and exhibits the thermally induced inversion of the cholesteric sense (Figure 5). This observation suggests that the aromatic-ring interaction between the solvent and the side-chain phenyl group is not essential for determining the cholesteric sense.

\section{REFERENCES}

1. A. Elliott and E. J. Ambrose, Discuss. Faraday Soc., 9, 246 (1950). 
2. a) C. Robinson, Trans. Faraday Soc., 52, 571 (1956); b) C. Robinson, J. C. Ward, and R. B. Beevers, Discuss. Faraday Soc., 25, 29 (1958); C. Robinson, Tetrahedron, 13, 219 (1961); d) C. Robinson, Mol. Cryst., 1, 467 (1966).

3. D. B. DuPré and R. W. Duke, J. Chem. Phys., 63, 143 (1975).

4. I. Uematsu and Y. Uematsu, Kobunshi, 25, 175(1976).

5. J. L. Fergason, N. N. Goldberg, and R. J. Nadalin, Mol. Cryst., 1, 309 (1966).

6. T. V. Samulski and E. T. Samulski, J. Chem. Phys., 67, 824 (1977).

7. D. B. DuPré, R. W. Duke, W. A. Hines, and E. T. Samulski, Mol. Cryst. Liq. Cryst., 40, 247 (1977).

8. G. D. Fasman, Ed., "Poly- $\alpha$-Amino Acids," Vol. 1, Marcel Dekker, New York N.Y., 1967.

9. J. B. Milstien and E. Charney, Biopolymers, 9, 991 (1970).
10. E. M. Bradbury, A. R. Downie, A. Elliott, and W. E. Hanby, Proc. R. Soc., London, Ser. A, 259, 110 (1960).

11. H. Toriumi, Y. Kusumi, Y. Uematsu, and I. Uematsu, Rep Prog. Polym. Phys. Jpn., 21, 569 (1978).

12. P. N. Keating, Mol. Cryst. Liq. Cryst., 8, 315 (1969).

13. E. M. Bradbury, B. G. Carpenter, and H. Goldman, Biopolymers, 6, 837 (1968).

14. K. Tsuji, H. Ohe, and H. Watanabe, Polym. J., 4, 553 (1973).

15. E. L. Wee and W. G. Miller, J. Phys. Chem., 75, 1446 (1971).

16. H. de Vries, Acta Cryst., 4, 219 (1951).

17. Y. Uematsu and I. Uematsu, "Mesomorphic Order in Polymers," ACS Symposium Series, No 74, American Chemical Society, Washington, D. C., 1978, pp 136156.

18. W. J. A. Goossens, Mol. Cryst. Liq. Cryst., 12, 237 (1971). 